\title{
Articular involvement in juvenile localized scleroderma
}

\begin{abstract}
The articular involvement (ArIn) of juvenile localized scleroderma was studied retrospectively in 190 patients with juvenile localized scleroderma patients. Of these ArIn was detected in 125 patients $(65,7 \%)$, among them $99 \%$ were in cases of linear and unilateral form of JLS. It was presented by joint pain in $25 \%$ of patients, limitation of joint movement in $62 \%$ of affected patients, mostly due to periarticular induration or tissue fibrosis, joint effusions - in 1\% of ArIn. $25 \%$ of affected children complained of joint pain. Limitation of joint movements was mostly (in $70 \%$ ) due to the induration of soft tissue and skin around the joint and was reversible under therapy. Joint effusions were observed in 1\% of ArIn. Ultrasound investigation imaging showed slight "subclinical" -synovitis and tenosynovitis in $80 \%$ of ArIn parients. Joint space narrowing by X-ray was detected in 12, articular erosions - in 2 children. In 1 girl with unilateral scleroderma MRI with contrasting detected avascular osteonecrosis of tibia. The levels of detected autoantibodies and markers of fibrosis were significantly higher in the patients with ArIn. All the patients with ArIn received immunodepressant therapy - glucocorticosteroids (GC) orally $1 \mathrm{mg} \backslash$ kilo for 6-8 weeks, then tapered and withdrawn through the 3-6 months, and methotrexate (MTX) parenterally $12 \mathrm{mg}$ sq.m. for at least 2 years. In $76 \%$ of cases of ArIn this treatment increased range of joint movements or stopped flexion contractures. Therapeutic efficacy was worse in prolonged JLS with ArIn.
\end{abstract}

Keywords: juvenile localized scleroderma, articular involvement, antibodies profile, X-ray, ultrasound changes, therapy
Volume 10 Issue 2 - 2018

\section{Osminina MK, Geppe NA, Shpitonkova OV}

Pediatric Department of Federal State Autonomous Educational Institution of Higher Education, I.M. Sechenov First Moscow State Medical University of the Ministry of Health of the Russian Federation, Russia

Correspondence: Osminina MK, Pediatric Department, IM Sechenov First Moscow State Medical University, Moscow, Russia,Email mk_osminina@mail.ru

Received: February 15, 2018 | Published: March 14, 2018
Abbreviations: JLS, juvenile localized scleroderma; JSS, Juvenile systemic sclerosis; ArIn, Articular involvement; $\mathrm{RF}$, rheumatoid factor; ESR, erythrocyte sedimentation rate; ANF, antinuclear factor; Cab, autoantibodies to collagen, CG, cryoglobulins;FN, serum fibronectine; HA, hyaluronic acid; GC, glucocorticosteroids, MTX, methotrexate

\section{Introduction}

Juvenile scleroderma is a rare disease of childhood. It includes two main clinical entities, juvenile systemic sclerosis (JSS) and juvenile localized scleroderma (JLS). Both have a common pathophysiology, with an initial inflammatory phase of the disease associated with endothelial disfunction, and a later fibrotic phase with skin thickening. In JSS many organs and systems may be affected - vascular (Vessels) (Raynaud phenomenon), cutaneous (Skin), gastrointestinal tract, pulmonary, cardiac and musculoskeletal system, while in JLS the process of fibrosis involves manly the skin and subcutaneous tissues. Articular involvement (ArIn) is the most frequent finding in JLS, patients who developed arthritis often have a positive rheumatoid factor (RF), and sometimes elevated erythrocyte sedimentation rate (ESR) and circulating antibodies. ${ }^{1}$

The pathogenesis of scleroderma and ArIn is compex and incompletely understood. Vascular injury, autoimmune dysfunction and connective tissue remodeling with excessive collagen production are the main pathways of the disease. Mechanism of fibrosis and the role of disease -specific autoantibodies have been extensively investigated. Antinuclear antibodies, RF, anti-double-stranded DNA antibodies and anti-histone antibodies were detected not only in the systemic, ${ }^{2}$ but also in the localized forms of scleroderma. ${ }^{3}$ Thus, according to some authors, the antinuclear factor is detected in 46$80 \%$, antidouble- stranded DNA antibodies - in $50 \%$, antihistone antibodies - in $47 \%$, rheumatoid factor - in $26 \%$ of adult patients with limited scleroderma. ${ }^{4}$ There are some data stating that antinuclear factor directly correlates with joint lesions. ${ }^{5}$ Patients with rheumatic conditions often have mixed cryoglobulins, composed of class $\mathrm{M}$ and rheumatoid factor immunoglobulins, class $\mathrm{G}$ polyclonal immunoglobulins and fibronectin. The level of cryoglobulins also correlates with clinical and laboratory activity of scleroderma.

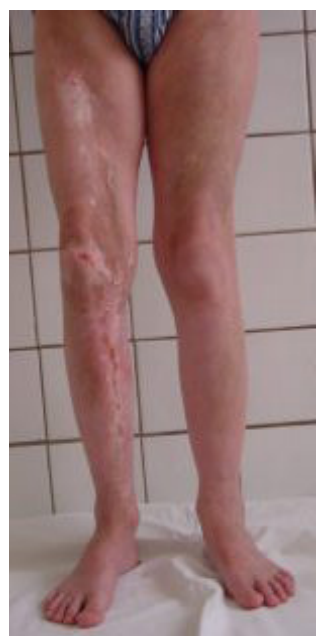

A

Figure I (A) Periarticular contracture. 
The extracellular matrix of skin, tendon and bonetissue is composed mainly of type I collagen, and of type III collagen to a lesser extent. Type II collagen is mostly located in the articular cartilage, collagen IV - in basal cell membranes. Some researchers detect type I and IV collagen molecule autoantibodies in systemic scleroderma. ${ }^{2}$ As the result of monocyte overproduction, an intensive secretion of monokines, such as fibronectin and interleukin-1 are stimulated. Fibronectin has a high affinity to the native and denatured collagen. Some data suggests that in scleroderma level of fibronectin and hyaluronic acid are increased, and could be used as biomarkers of fibrosis..$^{6,7}$

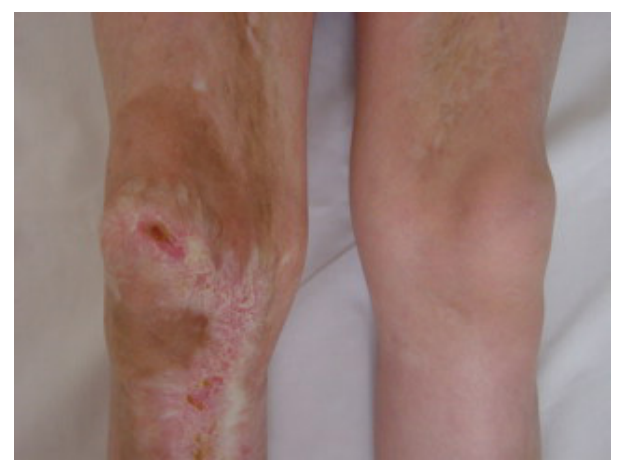

B

Figure I (B) Fibrotic skin lessions around the knee.

The articular manifestations present both in JSS and JLS, as far as in children the incidence of JLS is fourfold higher than JSS, the articular involvement (ArIn) in JLS represents a challenge to growth disturbances. ArIn lead to severe joint contractions, orthopedic deficits and motor disabilities. Arthritis occurs in $27.5 \%$ of patients in pediatric JSS cohort and in $17 \%$ of JLS patients, in comparison to $18 \%$ in adult systemic sclerosis cohort. ${ }^{8}$

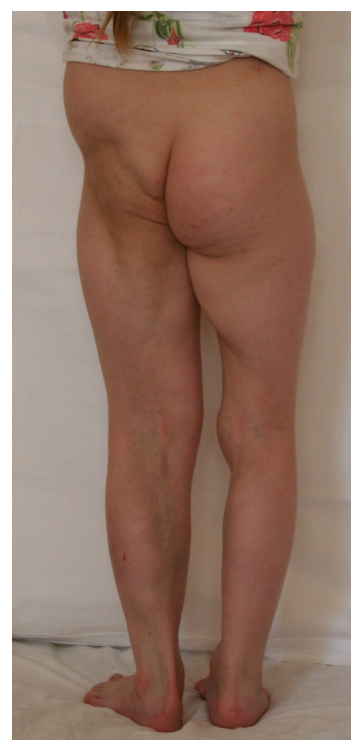

Figure 2 Pansclerotic scleroderma with articular involvement.

.Moreover, scleroderma affects children in the period of their intensive growth, so ArIn could cause extremity length differences, functional skeletal impairment, growth retardation of extremities (Figure 1) (Figure 2). Children with JLS and ArIn tend to have asymmetric joint involvement with no erosions, an accelerated course with predominant musculoskeletal disease and rapid development of contractures (Figure 3).

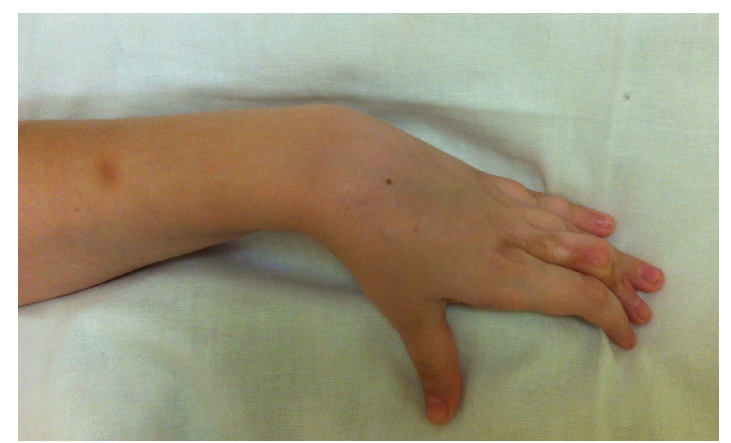

Figure 3 Flexion contracrutes in lineal scleroderma of hand.

ArIn mostly develops in linear and deep subtypes of JLS. There is a unilateral subtipe, which usually begins in childhood and results in severe deformities of extremities (Figure 4). The origin of ArIn in JLS may be of different types: caused by scleroderma changes (induration or fibrosis) in skin and periarticular tissues, arthritis itself, avascular necrosis of joints and bones. Modern techniques such as ultrasound and magnetic resonance imaging are used now in pediatric rheumatology to describe structural articular lesions at the early stages of JLS.

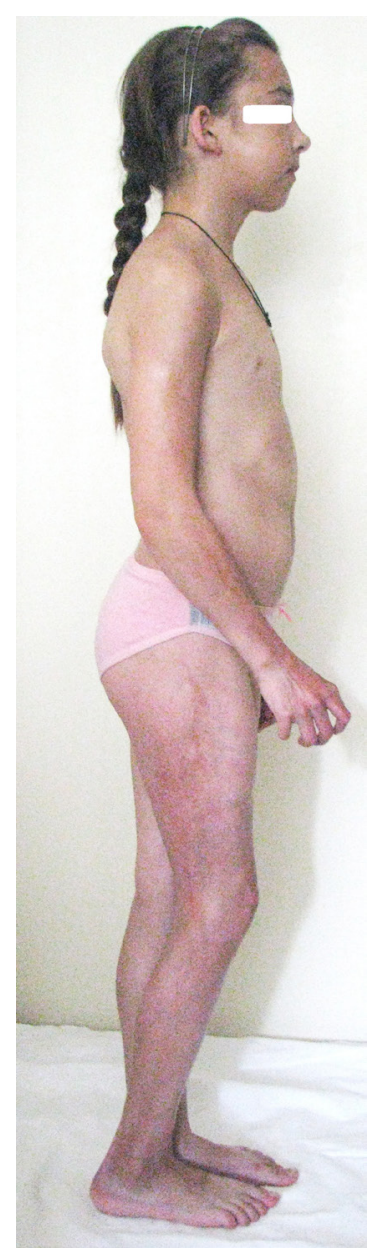

Figure 4 Unilateral scleroderma. 


\section{Materials and methods}

The ArIn was studied retrospectively in 190 children with JLS, girls -128 , boys-62), who were under supervision at the specialized rheumatology department of I.M. Sechenov First. Moscow Medical University for a five - year period from 2006-2011. The surveyed group consisted of children from 3 to 17 years of age, including 128 girls and 62 boys ( $\mathrm{F} \backslash \mathrm{M}$ ratio 1:2). The patients with JLS were divided into groups based on the clinical forms of the disease in accordance with the preliminary classification criteria. ${ }^{9}$ At the time of examination the patients with different clinical forms of the disease had dissimilar disease duration and activity, $65 \%$ of all the patients did not receive therapy with immunodepressants.

A history of joint manifestations was obtained by standard physical examination, X-ray, MRI ( in some cases), common blood tests, antinuclear antibodies (antinuclear factor (ANF), rheumatoid factor (RF), antitopoisomerase 1 and anticentomere antibodies,antibodies to DNA, autoantibodies to collagen (Cab) types I-IV, cryoglobulins (CG), serum fibronectine (FN) and hyalyronic acid (HA) levels. Levels of Scl-70 and anti-centromere antibodies were measured by enzyme immunoassay test using kit "Anti-Scl-70 Orgentec", (Germany). ANF was determined by indirect immunofluorescence. RF was determined by ELISA test with the normal values of $<20 I U / m L$, and anti- DNA was determined by ELISA test with the refference value of $<20 I \mathrm{U} / \mathrm{mL}$. Unconjugated concentration of FN was measured by ELISA ("Fibronectin Technoclone", Austria). Normal values of unconjugated FN are 70 to $148 \mathrm{mg} / \mathrm{ml}$ according to the manufacturer's recommendations. The concentration of HA in blood serum was measured by ELISA testing using "HA Testkit Corgenix", (USA). The level of HA in children was considered to be high when its concentration exceeded $30 \mathrm{ng} / \mathrm{ml}$ in accordance with the recommendations of the manufacturer. The presence of cryoglobulins in blood serum was determined with "Solar" PV 1251C (Solar, Belarus) spectrophotometer at a wavelength of $500 \mathrm{~nm}$ in accordance with the optical fluid density difference in a buffer solution $(\mathrm{pH} 8.6)$, incubated for 1 hour at the temperature of $4^{\circ} \mathrm{C}$ and then at $37^{\circ} \mathrm{C}$. The normal values are up to 0.06 optical density. Determination of type I-IV collagen antibodies in blood serum was performed with ELISA method.The test was considered positive if the negative control value exceeded. To interpret the results of the research the concept of "positive" was introduced for this test. The results were thought to be "positive" if they showed exceeding reference values in the tests with quantitative expression, presence of Scl-70 and anticentromere antibodies in blood serum, antinuclear factor titer of 1:80 or higher.

Statistical analysis of the results was performed with the use of Statistica 6.0 software. The quantitative indices were presented as mean values \pm standard deviation and range of values. Quality indices were presented as an absolute number of observations and proportion (in \%) of the total number.The validity of differences in the compared values was determined by Student's t-test for interval variables. The differences were considered statistically significant at $\mathrm{p}<0.01$.

\section{Results and discussion}

According to the clinical form of JLS the patients were divided into 4 groups: 14 patients with plaque morphea, 89- linear scleroderma, 77 - generalized morphea (including 31 with unilateral form), pansclerotic in 3 patients, mixed morphea - in 7 patients (Table 1).

Mean age of the disease onset was seven years and six months (range 18 months -15.5 years). As the Table 1 shows, the vast majority of surveyed patients were children with severe forms of JLS - linear scleroderma of limbs, generalized morphea (including unilateral form) and pansclerotic scleroderma. ArIn was noticed in 125 patients $(65.7 \%)$, among them the $99 \%$ was in cases of linear and unilateral form of JLS. It was presented by joint pain in $25 \%$ of patients, limitation of joint movement in $62 \%$ of affected patients, mostly due to periarticular induration or tissue fibrosis, joint effusions - in $1 \%$ of ArIn. Significantly more frequently joints were affected in linear scleroderma of extremites and unilateral form. In linear scleroderma of head, contracture of temporo-mandibular joint was only in 1 case, with prolonged disease duration, for more than 10 years.

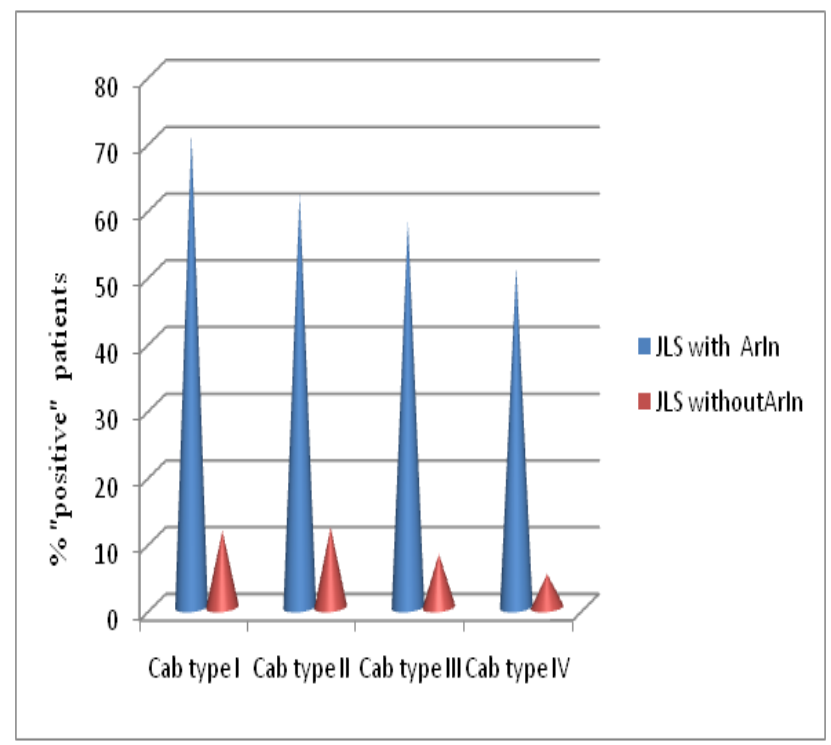

Figure 5 Detection rate of antibodies to type I-IV collagens in patients with Arln and without Arln.

We did not find significant blood leucocytosis or elevated ESR in our series. The analysis of the data suggests that patients in both groups were "positive" for most of the required tests, except sclerodermospecific antibodies. At the same time, the absolute percentage of positive values was higher in the group I - children with ArIn. (Differences are statistically valid) (Figure 2).

The cohort study of patients showed that ANF was detected in $56 \%$ and RF in $28.4 \%$ of patients with ArIn. The obtained data are compatible with the results of the international multicenter studies of children, in which the proportion of ANF detection in children with JSS ranges from $81 \%$ to $97 \%{ }^{8}$ and it amounts to $42.3 \%$ in patients with JLS. ${ }^{9,10}$ It is known, that in scleroderma the presence ANF and RF correlates with articular manifestations of the disease. RF has been detected, as low titre, in $16 \%$ of the patients who had JLS, and significantly correlated with the presence of arthritis. ${ }^{1}$ In adults, Ig M RF is present in $30 \%$ of patients who have LS, particularly who have GM, and seems to be correlated with disease severity. ${ }^{11}$ According to the literature data, there is no connection between antinuclear factor detection and any clinical form of JLS. ${ }^{5}$

In our series sclerodermospecific antibodies and ds-DNA were detected in small percentage of ArIn patients and in none without it. It is no wonder, because these antibodies are mainly found in JSS. Surprisingly, the levels of CG and collagen antibodies were elevated in patients with ArIn. CG was the third most frequently detected element $(27.6 \%)$ in JLS with ArIn, while its detection rate was less than $5.2 \%$ in patients without ArIn. Increased level of CG in ArIn 
patients confirms the role of immune mechanisms in the pathogenesis of this clinical form. ${ }^{12}$ Similar to it, the levels of FN and HA in serum had a higher detection rate in children with articular manifestations.
Summing it up, the levels of autoantibodies and markers of fibrosis were significantly higher in the patients with ArIn (Figure 5).

Table I Articular involvement in different clinical subtypes of juvenile localized scleroderma

\begin{tabular}{|c|c|c|c|}
\hline \multicolumn{2}{|c|}{ Clinical subtypes of juvenile localized sclerodema } & \multirow{2}{*}{$\begin{array}{l}\text { Number of patients } \\
14\end{array}$} & \multirow[t]{2}{*}{ Articular involvement (\%) } \\
\hline & Plaque morphea & & \\
\hline JLS & -superficial & 4 & \\
\hline \multirow{2}{*}{$(N=190)$} & - deep & 10 & 1 \\
\hline & Linear scleroderma & 89 & \\
\hline \multirow{2}{*}{ Male -62} & - trunkllimbs & 58 & 97 \\
\hline & - head & 31 & 3 \\
\hline Female - 128 & Generalized morphea(-unilateral form *) & $77(31 *)$ & $26(100 *)$ \\
\hline \multirow[t]{2}{*}{$(\mathrm{N}=32)$} & Pansclerotic scleroderma & 3 & 100 \\
\hline & Mixed morphea & 7 & 20 \\
\hline
\end{tabular}

Table 2 Serum antibodies profile in JSL patients with and without Arln

\begin{tabular}{|c|c|c|c|c|c|}
\hline \multicolumn{2}{|l|}{ Indices } & $\begin{array}{l}\text { The range of } \\
\text { absolute value }\end{array}$ & $\begin{array}{l}\text { Mean level }(M \pm M) \\
\text { JLS pts }(N=190)\end{array}$ & $\begin{array}{l}\% \text { of positive in JLS } \\
\text { pts with Arln (group } \\
\text { IN=I25) }\end{array}$ & $\begin{array}{l}\% \text { of positive in JLS pts } \\
\text { without Arln (group II } \\
N=65 \text { ) }\end{array}$ \\
\hline \multicolumn{2}{|c|}{ Antinuclear antibodies* (titer) } & $\mathrm{I}: 80-\mathrm{I}: 640$ & - & 56 & 3,2 \\
\hline \multicolumn{2}{|c|}{ Rheumatoid factor*(IU/ml) } & $11-125$ & $82,4 \pm 60,3$ & 28,4 & 2,1 \\
\hline \multicolumn{2}{|c|}{ ds-DNA (IU/ml) } & $0-62$ & $34,5 \pm 3,6$ & 2,5 & - \\
\hline \multicolumn{2}{|c|}{ Anti Scl-70 antibodies } & - & - & 8,5 & - \\
\hline \multicolumn{2}{|c|}{ Anti-centromere B antibodies } & - & - & 0,5 & - \\
\hline \multicolumn{2}{|c|}{$\begin{array}{l}\text { Cryoglobulins (optical density } \\
\text { unit)* }\end{array}$} & $0,037-0,216$ & $0,05 I \pm 0,037$ & 27,6 & 5,2 \\
\hline \multirow{4}{*}{ 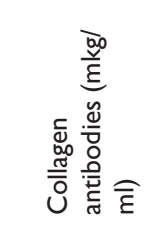 } & I type* & $0,297-0,893$ & $0,405 \pm 0,112$ & 71 & $\mathrm{II}, 5$ \\
\hline & II type* & $0,330-1,129$ & $0,55 I \pm 0,16 I$ & 62 & 12 \\
\hline & III type* & $0,287-0,681$ & $0,370 \pm 0,108$ & 58 & 8 \\
\hline & IV type & $0,151-0,577$ & $0,300 \pm 0,085$ & 51 & 5 \\
\hline \multicolumn{2}{|c|}{ Serum fibronectin* (mg/ml) } & $68-264$ & $|24,8+4|, 9$ & 46,5 & 14 \\
\hline \multicolumn{2}{|c|}{ Hyaluronic acid* (ng/ml) } & $5,4-68,4$ & $15,7 \pm 16,7$ & 23,7 & 8,5 \\
\hline
\end{tabular}

Ultrasounography showed synovitis or tenosynovitis. Radiologic abnormalities in knee, hip, ankle and wrist joins were seen 8 patients with linear JSL, 5 with unilateral generalized morphea and 1 - pansclerotic morphea. In 12 children joint space narrowing was detected, in 2- articular erosions. Patients with articular erosions had prolonged disease duration (more than 7 years) and were treated unadequate. MRI with contrasting visualised avascular osteonecrosis of tibia in 1 girl with unilateral scleroderma.

All the patients with ArIn received immunodepressant therapy glucocorticosteroids (GC) orally $1 \mathrm{mg} \backslash$ kilo for 6-8 weeks, then tapered and withdrawn through the 3-6 months, and methotrexate (MTX) parenterally $12 \mathrm{mg} \backslash \mathrm{sq} . \mathrm{m}$. for at least 2 years. In $76 \%$ of cases of ArIn this treatment increased range of joint movements or stopped flexon contractures. Therapeutic efficacy was worse in prolonged JLS with ArIn, in case of 5 and more years without treatment.

\section{Conclusion}

ArIn in JLS influences greatly the outcome of the disease and social adaptation of children. The frequency of occurance of ArIn in JSL has been previously reported. In the present series of 190 JSL chidren, ArIn was detected in $65.7 \%$ of cases, mainly in linear and unilateral form of JLS. Only $25 \%$ of affected children complained of joint pain, tenderness, or pain on joint motion. Flexion contractures of joints with disability were the main complaint. Limitation of joint movements was mostly (in 70\%) due to the induration of soft tissues and skin around the joint and was reversible under therapy. In the contrary to lower percentage of joint effusions - in 1\% of ArIn. Ultrasound investigation of affected joints showed slight "subclinical" -synovitis and tenosynovitis in $80 \%$ of ArIn parients. While join space narrowing by X-ray was detected in 12, articular erosions - in 2 children. In 1 girl with unilateral scleroderma MRI with contrastingdetected avascular osteonecrosis of tibia. 
In our series the levels of detected autoantibodies and markers of fibrosis were significantly higher in the patients with ArIn. The detection of autoantibodies and markers of fibrosis involved in the pathogenesis of JLS is an evidence of systemic autoimmune and collagen synthesis disturbances in these patients and also determines the target points for the treatment with immunosuppressants and antifibrotic agents. According it JLS with ArIn of numerous joints could be considered as systemic form of scleroderma. Especially, it is true for the unilateral form JLS, that usually starts in childhood and leads to disability and impaired quality of life.

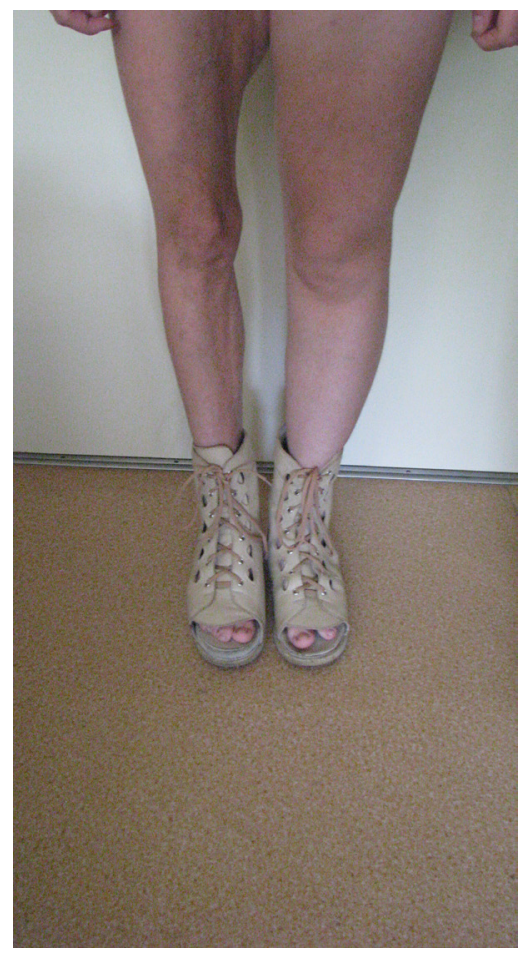

Figure 6 Orthopedic correction of leg deformity.

Contractures in JLS are mainly reversible thanks to reversibility of oedema and induration stages of scleroderma under treatment with GC and the* partly reversible (fibrotic lesions)*, while treated with MTX. Only few patients had irreversible arthritis with joint space narrowing and erosions. In JSS clinicians rarely doubt about the decision of including GC and cytotoxic drugs in the therapy. In contrast, the treatment of LJS is believed to have successful outcomes with the local use of immunosuppressive agents.

It should be emphasized that the chronic nature of scleroderma, the prevalence of linear form of the disease in children, complicated by irreversible fibrosclerotic defects of the musculoskeletal system, growth retardation of extremites encourage pediatric rheumatologists to elaborate a unified treatment protocol with GC, MTX or other antifibrotic drugs. There are few reports of successful treatment with biologic agents in resistant cases. ${ }^{13,14}$ The appropriate treatment must be started at early stages of JLS, to prevent joint contractures and severe deformities of legs and hands. Additionaly patients with ArIn need orthopedic correction (Figure 6), reconstructive surgery in adults (arthrodesis and flap surgery).

\section{Acknowledgements}

None.

\section{Conflict of interest}

Authors declare there is no conflict of interest in publishing the article.

\section{References}

1. Baron M, Lee P, Keystone E. The articular manifestations of progressive systemic sclerosis. Annals of the Rheumatic Diseases, 1982;41:147-152.

2. Kayser C, Fritzler MJ. Autoantibodies in systemic sclerosis: unanswered questions. Front Immunol. 2015;6:167-175.

3. Arkachaisri T, Fertig N, Pino S, et al.Serum autoantibodies and their clinical associations in patients with childhood- andadult-onset linear scleroderma. A single-center study. J Rheumatol. 2008;35(12):24392444.

4. Chung L, Lin J, Furst DE, et al. Systemic and localized scleroderma. Clin Dermatol. 2006;24(5):374-392.

5. Gilliam AE, Gilliam AC. Localized forms of scleroderma. Scleroderma: from Pathogenesis to Comprehensive Management. John Varga, Christopher P Denton, Fredrick M Wigley, editors. GerSpringer Science+Business Media LLC, New York; 2012. p. 113-123.

6. Varga J, Denton ChP, Wigley FM. Mechanism of fibrosis. Scleroderma: from Pathogenesis to Comprehensive Management. Springer Science+Business Media LLC, New York; 2012. p. 225-265.

7. Yoshizaki A, Iwata Y, Komura K, et al. Clinical significance of serumhyaluronan levels in systemic sclerosis: association with disease severity. J Rheumatol. 2008;35(9):1825-1829.

8. Foeldvari I. Juvenile systemic sclerosis. Scleroderma: From Pathogenesis to Comprehensive Management. John Varga, Christopher P Denton, Fredrick M Wigley, editors. Springer Science+Business Media LLC, New York; 2012. p. 93-100.

9. Zulian F. Juvenile localized scleroderma. Scleroderma: From Pathogenesis to Comprehensive Management. John Varga, Christopher P Denton, Fredrick M Wigley, editors, Springer Science+Business Media LLC, New York, 2012. p. 85-92.

10. Osminina MK, GeppeNA, Tougarinova GV, et al. The New Armenian Medical Journal, 2016;10(4):13-21.

11. Martini G, Foeldvari I, Russo R, et al. Systemic sclerosis in childhood: clinical and immunologic features of 153 patients in an international database. Arthritis Rheum. 2016;54(12):3971-3978.

12. Takehara $\mathrm{K}$, Sato $\mathrm{S}$. Localized scleroderma is an autoimmune disorder. Rheumatology (Oxford).2005;44(3):274-279.

13. Elhai M, Meunier M, Matucci-Cerinic M, et al. Outcomes of patients with systemic sclerosis associated polyarthritis and myopathy treated with tocilizumab or abatacept: a EUSTAR observational study. Curr Opin Rheumatol. 2011;23(3):273-277.

14. Martini G, Campus S, Raffeiner B, et al. Tocilizumab in two children with pansclerotic morphoea: a hopeful therapy for refractory cases? Clin Exp Rheumatol. 2017;35 Suppl 106(4):211-213. 